Revue d'histoire de l'enfance « irrégulière »

Le Temps de l'histoire

11 | 2009

Paroles libres, paroles captives

\title{
Pâtés et tâches à la plume Sergent-Major : les trésors enfouis des cahiers d'écoliers de jeunes délinquants
}

Mathias Gardet

\author{
(2) OpenEdition \\ Journals \\ Édition électronique \\ URL : http://journals.openedition.org/rhei/3074 \\ DOI : 10.4000/rhei.3074 \\ ISBN : 978-2-7535-1650-2 \\ ISSN : $1777-540 \mathrm{X}$ \\ Éditeur \\ Presses universitaires de Rennes

\section{Édition imprimée} \\ Date de publication : 1 octobre 2009 \\ ISBN : 978-2-7535-0927-6 \\ ISSN : $1287-2431$
}

Référence électronique

Mathias Gardet, «Pâtés et tâches à la plume Sergent-Major : les trésors enfouis des cahiers d'écoliers de jeunes délinquants », Revue d'histoire de l'enfance « irrégulière » [En ligne], 11 | 2009, mis en ligne le 01 octobre 2011, consulté le 04 décembre 2020. URL : http://journals.openedition.org/rhei/3074 DOI : https://doi.org/10.4000/rhei.3074

Ce document a été généré automatiquement le 4 décembre 2020.

(C) PUR 


\title{
Pâtés et tâches à la plume Sergent- Major : les trésors enfouis des cahiers d'écoliers de jeunes délinquants
}

\author{
Mathias Gardet
}

1 J'ai eu la chance d'avoir accès à un volume très conséquent de dossiers (plus de 15.000) de jeunes placés dans le centre public d'observation de Savigny-sur-Orge, une des premières institutions créées par la toute jeune direction de l'Education surveillée du ministère de la Justice en $1945^{1}$. Dans chacun de ces dossiers très épais, malgré la brièveté du séjour dans le centre (trois mois), j'ai découvert un à deux petits cahiers d'écoliers ; un corpus donc exceptionnel si l'on se réfère à ceux existant dans le monde scolaire. ${ }^{2}$ À part quelques exercices typiquement scolaires: dictées, problèmes, questions d'histoire sommaires ou cartographie de la France, ces cahiers sont surtout composés de rédactions et de dessins, exercices auxquels les jeunes ont été familiarisés durant leur scolarité primaire, même si certains sujets ont été détournés et ajustés au contexte de la prise en charge judiciaire : «Ecris au juge pour lui dire ce que tu penses de ton placement", "Supposes que pour une journée on te nomme à la place d'un éducateur, qu'est-ce que tu fais?», "Dis-moi tout ce qui te passe par la tête sans réfléchir», "Raconte la journée d'un agent de police», "Si tu avais le pouvoir de te transformer en animal, qu'est-ce que tu choisirais et pourquoi?», «Raconte une journée que tu aimerais vivre », «Si tu étais invisible, qu'est-ce que tu ferais?», «Tu viens de gagner 10 millions au loto qu'est-ce que-tu fais?", «Tu vois un lion dans une cage, à quoi rêve-t-il ? ", "Si le monde devait disparaître dans deux jours, que feraistu ?» ... ou bien dessine « comment tu t'imagines à 30 ans, à 45 ans, à 60 ans », « les six principaux événements de ta vie ", «les événements heureux et malheureux », «ton dernier dimanche de liberté », « une journée que tu aimerais vivre »...

2 Malgré l'adoption d'une forme traditionnelle et très ancienne (remontant au moins au XVIIIe siècle) qu'est la rédaction ${ }^{3}$, il ne s'agit pourtant pas d'un exercice scolaire. Même 
si, le support choisi pour les conserver, ces petits cahiers, format $17 \mathrm{~cm} \mathrm{X} 22 \mathrm{~cm}$, de la marque "Clair matin " ou bien "Hermès " ou bien encore "Chambord", avec leurs pages à grands carreaux, une ligne rouge sur la gauche pour démarquer la marge permettant aussi de laisser une place pour les annotations en rouge du maitre; des cahiers couverts de cette écriture arrondie et méticuleuse ou au contraire maladroite avec des fautes d'orthographes, des pâtés, des gribouillages sous l'effet de la plume Sergent-Major - remplacée à partir des années 1960 par l'écriture plus brouillonne du stylo à bille - ou encore avec des dessins naïfs ou habilement croqués, d'un impact visuel très fort, présentés de façon libre ou bien plus formelle en une bande dessinée de six petits cadres de $8 \mathrm{~cm}$ de côté, nous renvoient à notre propre enfance d'élèves sur les bancs de l'école. Les jeunes placés au centre de Savigny ont tous plus de 13 ans et échappent donc jusqu'à la fin des années 1960 à l'obligation scolaire (le prolongement de la scolarité de 14 à 16 ans édictée avec la réforme Bertouin ne prend effet que pour la génération des jeunes ayant eu plus de 13 ans en 1967). Le personnel qui les encadre pendant leur court séjour à Savigny n'est donc pas issu de l'Education nationale mais de la Justice : éducateurs formés dans des écoles privées dès le début des années quarante puis dans la première école publique de Vaucresson à partir de 1954, puis dans l'annexe de Savigny-sur-Orge à partir des années 1960 (des préfabriqués accolés au centre d'observation). Il serait ainsi possible d'y détecter une forte influence des méthodes d'éducation nouvelle ou de pédagogies actives, s'il n'y avait eu l'action d'un stylo rouge soulignant la récurrence de certains mots et annotant en marge quelques commentaires elliptiques mais non moins parlants. Il ne faut pas s'y méprendre, nous sommes loin de l'action d'un pédagogue éclairé bénéficiant, comme cela a été parfois le cas dans certains centres de rééducation, d'une plus grande marge de manœuvre vis-àvis des programmes en vigueur - jeunes inadaptés obligent -, mais bien face à une nouvelle modalité de tests psychologiques orchestrés par le médecin psychiatre, venant compléter la vaste batterie de tests à laquelle sont soumis les jeunes durant leur séjour.

Qui dit obligation et espace disciplinaire, voire même enfermement, ne veut cependant pas dire forcément paroles captives, tronquées ou langue de bois. Comme l'avait déjà démontré l'historien Jean-Claude Vimont dans son étude sur la colonie pénitentiaire pour garçons des Douaires (dans l'Eure) ${ }^{4}$, les graffitis retrouvés sur les murs des cellules ne sont pas la seule expression d'une violence ou d'un formatage institutionnels, mais bien un lieu de prise de parole et de communication. Les jeunes détenus y racontent certes leurs conditions de vie dans l'établissement ou expriment des propos revanchards contre l'ordre établi, mais aussi y impriment leurs codes et positionnement au sein de leur bande, leurs faits et gestes avant leur arrestation et s'échangent des informations. De la même façon, les jeunes de Savigny astreints à l'écriture et au dessin durant des après-midi entiers, finissent par s'emparer de ce moyen d'expression pour se raconter, évoquer leurs ressentis et leurs opinions avec une grande liberté. Ils le font d'autant plus facilement que les sujets des rédactions et dessins ne ressemblent pas à un interrogatoire, mais font plutôt appel à l'imaginaire. En ce sens la méthode projective inventée par les psychiatres et psychologues se révèle très efficace, même si le but poursuivi par ces derniers n'est pas de susciter un aveu.

4 L'engrainage judiciaire et l'expertise auxquels ces jeunes sont soumis à un moment donné de leur existence constituent une opportunité pour l'historien en créant l'archive et rendant bavards des gens qui normalement laissent peu de trace. Ce support si familier des cahiers scolaires, nous invite de façon troublante à dépasser la fixation sur le fait délictuel si prégnante dans ces dossiers, pour regarder et écouter ces 
jeunes sous un autre jour, pour se laisser emporter par ces récits et cet imaginaire propre à l'enfance, qui mélangent une vision acérée sur leur réalité et les rêves. Ces textes et dessin ne nous offrent-ils pas ainsi d'autres pistes pour la recherche?

Pour une histoire de la minorité et de la majorité qui ne se limiterait pas aux débats et batailles législatives, mais à la façon dont ces seuils d'âges sont perçus par les jeunes mineurs eux-mêmes?

6 Pour une histoire de la scolarité des jeunes, avec pendant longtemps l'enjeu du CEP, comme rite de passage décisif pour acquérir une certaine autonomie?

7 Pour une histoire de la guerre vécue par les jeunes, ces enfants de la guerre qui se retrouve souvent à faire la guerre (Indochine) ?

8 Pour une histoire de l'immigration et en particulier de l'immigration maghrébine en métropole (avec en filigrane une histoire de la guerre d'Algérie), en mettant en lumière le phénomène des jeunes dits "isolés" souvent absents des statistiques migratoires?

Pour une histoire des sociabilités de jeunes et de leurs loisirs, vues par eux-mêmes, phénomène antérieur à celui des Blousons noirs (1959-1964) qui les cristallisent autour de la notion de bande?

Pour une histoire de l'homosexualité et du regard porté par la société sur ce phénomène considéré comme déviant et plus généralement de la sexualité des jeunes?

Pour une histoire du travail des jeunes, de l'autonomie des jeunes dès l'âge de 13-14 ans, avec comme corollaire une histoire de l'apprentissage et du rapport au CAP ?

Pour bien d'autres histoire encore...

\section{NOTES}

1. Mathias Gardet, "Les écrits des jeunes mineurs délinquants : une revanche par l'image ", Paris, Vie sociale, $\mathrm{n}^{\circ} 1,2005$, pp. 73-78; «Les dossiers de jeunes délinquants. Uncorpus inédit aux défis de l'histoire", in Ludivine Bantigny, Jean-Claude Vimont, Les dossiers de personnalité. Sources et objets de l'histoire, Rouen, Presses universitaires de Rouen, (à paraître 2009).

2. André Chervel, « les travaux d'élèves » chapitre V de l'Histoire de l'enseignement, XIXe-XXe siècles. Guide du chercheur, 2e éd. (sous la direction de Thérèse Charmasson), Paris, Institut national de recherche pédagogique, Comité des travaux historiques et scientifiques, novembre 2006, pp. 601-624

3. Brigitte Dancel, Un siècle de rédactions : Ecrits d'écoliers et de collégiens, Grenoble : CRDP de l'académie de Grenoble, 2001, 264 p.

4. Jean-Claude Vimont, "Les graffitis de la colonie pénitentiaire des Douaires ", dans Frédéric Chauvaud, Jacques-Guy Petit (dir.), L'histoire contemporaine et les usages des archives judiciaires (1800-1939), Histoire et archives, hors série $n^{\circ} 2$, Paris, Honoré Champion Editeur, 1998, p. 139-153. 
INDEX

Index géographique : Savigny-sur-Orge

Mots-clés : écriture, rédactions, sciences psychiques

Index chronologique : $1945-1975$

\section{AUTEUR}

\section{MATHIAS GARDET}

Historien, maître de conférences en sciences de l'éducation, Université de Paris 8, Centre interdisciplinaire de recherche, culture, éducation, formation, travail (CIRCEFT) 